JORNADAS

\title{
MANEJO DEL CONTROL GLUCÉMICO: OBJETIVOS DE TRATAMIENTO Y MÉTODOS. HEMOGLOBINA GLICOSILADA A1C (HBA1C). FRUCTOSAMINA. AUTOMONITOREO GLUCÉMICO. GLUCOSURIA. CETONURIA
}

\author{
MANAGEMENT OF GLYCEMIA MONITORING: OBJECTIVES OF TREATMENT \\ AND METHODS. GLYCOSILATED HEMOGLOBIN A1C (HBA1C). FRUCTOSAMINE. \\ SELF-BLOOD GLUCOSE MONITORING. GLYCOSURIA. KETONURIA \\ Natalia Lueje
}

\section{RESUMEN}

En la medición estándar del control y el seguimiento en pacientes con diabetes, la hemoglobina glicosilada $(\mathrm{HbA} 1 \mathrm{C})$ presenta dificultades en la insuficiencia renal, en la cual puede no ser buen indicador del control glucémico. La fructosamina no es válida cuando la albumina es menor a $3 \mathrm{mg} / \mathrm{dl}$, mientras que la glucosuria y la cetonuria no son herramientas de medición efectiva en enfermedad renal. El automonitoreo glucémico (AMG) individualizado es un método útil en todos los estadios renales y el de mayor valor en el control y seguimiento en insuficiencia renal avanzada y tratamientos renales sustitutivos.

Palabras claves: objetivos de control, control metabólico, hemoglobina glicosilada A1c, automonitoreo glucémico.

Revista de la Sociedad Argentina de Diabetes 2017; Vol. 51 (81-85)

\section{ABSTRACT}

The standard measurement of control and follow-up in patients with diabetes, the glycosylated hemoglobin (HbA1C), present difficulties in renal insufficiency, in which it may not be a good indicator of glycemia monitoring. Fructosamine is not valid when albumin is less than $3 \mathrm{mg} / \mathrm{dl}$, whereas glucosuria and cetonuria are not effective measurement tools in the kidney disease. The individualized self-monitoring of blood glucose (SMBG) is a useful method in all kidney stages and of greater value in the control and follow-up in advanced renal insufficiency, and alternative kidney treatments.

Key words: objectives of control, metabolic control, glycosylated hemoglobin A1c, self-blood glucose monitoring.

Revista de la Sociedad Argentina de Diabetes 2017; Vol. 51 (81-85)
Especialista en Nutrición; Hospital Cetrangolo, Vicente López, Prov. de Bs. As.; miembro del Comité de Nefropatía de la Sociedad Argentina de Diabetes, CABA, Argentina

Contacto de la autora: Natalia Lueje

E-mail: na.lueje@hotmail.com
Correspondencia: Santiago del Estero 5579 (1605), Prov. de Bs. As., Argentina

Tel: (011) 4735-2545

Fecha de trabajo recibido: 18/08/17

Fecha de trabajo aceptado: 30/08/17

Conflictos de interés: la autora declara que no existe conflicto de interés

\section{Control glucémico}

La hemoglobina glicosilada ( $\mathrm{HbA} 1 \mathrm{c})$ es la medición estándar en pacientes con diabetes. Según las guías Kidney Disease Improving Global Outcomes (KDIGO) se recomienda una HbA1c menor a 7\% para prevenir o retardar la progresión de la enfermedad renal. Por otro lado hay que considerar que la mayoría de los ensayos realizados en pacientes con diabetes mellitus (DM) tipo 1 ó 2 ha excluido a aquellos que presentan marcada alteración de la función renal. En estos casos KDIGO recomienda no tratar a pacientes con riesgo de hipoglucemia a valores $\mathrm{HbA} 1 \mathrm{c}$ menor de $7 \%$ y se sugiere un valor mayor a $7 \%$ en los pacientes con múltiples comorbilidades y/o expectativa de corta vida; en un artículo de revisión se establece un límite superior de HbA1c menor a $8,5 \%$. Por ende, se sugiere individualizar los objetivos acorde a la situación clínica de cada 
paciente, a medida que avanza de estadios iniciales de enfermedad renal hasta los terminales sustitutivos o de trasplante renal ${ }^{1,2}$.

Estudios como el Diabetes Control and Complications Trial (DCCT) y el United Kingdom Prospective Diabetes Study (UKPDS) demostraron que los niveles de HbA1c predicen el riesgo de complicaciones microvasculares y el control estricto de la glucemia reduce el riesgo de desarrollo de albuminuria y la progresión del daño renal ${ }^{3,4}$.

La Asociación Americana de Diabetes ha recomendado a la población general de pacientes con diabetes valores de $\mathrm{HbA} 1 \mathrm{c}<7 \%$ y objetivos menos estrictos, en valores de $\mathrm{HbA} 1 \mathrm{c}<8 \%$, para pacientes con severas hipoglucemias o complicaciones avanzadas microvasculares y/o macrovasculares ${ }^{5}$.

Los pacientes con diabetes y declinación de la función renal presentan mayor riesgo de hipoglucemia debido a múltiples factores. El grupo de mayor riesgo lo constituyen los pacientes de edad avanzada; hay pocos datos respecto de los valores objetivos de $\mathrm{HbA} 1 \mathrm{c}$ en esta subpoblación de pacientes ${ }^{6,7}$.

Un estudio observacional de pacientes con enfermedad renal crónica (ERC), con filtrado glomerular $<60 \mathrm{ml} / \mathrm{min} / 1,73 \mathrm{~m}^{2}$ encontró una relación en " $U$ " entre mortalidad y $\mathrm{HbA} 1 \mathrm{c}$, con mayor mortalidad con $\mathrm{HbA} 1 \mathrm{c}>9 \%$ y menor de $6,5 \%$. Una forma de curva similar se observó en pacientes que realizaban hemodiálisis y diálisis peritoneal $\left.\right|^{8,9,10}$.

La presencia de enfermedad renal afecta los riesgos y beneficios del control glucémico estricto así como también condiciona los fármacos hipoglucemiantes a utilizar. En el estudio Action to Control Cardiovascular Risk en Diabetes (ACCORD), los efectos adversos del control intensivo con cifras de $\mathrm{HbA} 1 \mathrm{c}$ cercanas a lo normal aumentaron la mortalidad, probablemente por hipoglucemia, lo cual se vio incrementado en pacientes con enfermedad renal ${ }^{11,12}$.

El control intensivo de la glucemia se vería reflejado en mejoras del filtrado glomerular a los dos años en diabetes tipo 2 y a 10 años en diabetes tipo $1^{13}$.

\section{Métodos de medición}

Hemoglobina glicosilada (HbA1c)

La glucosa reacciona con el extremo amino terminal -valina- de la cadena $\mathrm{B}$ de la $\mathrm{HbA}$ resultando en la hemoglobina glicosilada A1C -HbA1c-, la cual es determinada actualmente para el seguimiento del control glucémico de los pacientes con diabetes.

Al evaluar este método en el seguimiento de los pacientes con insuficiencia renal existen algunas dificultades en el uso de la misma como proteína índice debido a que en el ambiente urémico existen varios factores que alteran su correlación con la glucemia y la de cualquier proteína plasmática glicosilada por vía no enzimática. La magnitud de la glicosilación está influenciada por la concentración de glucemia plasmática y la vida media de la hemoglobina en la cual la $\mathrm{HbA} 1 \mathrm{c}$ reflejaría la concentración de las glucemias en las ocho a 12 semanas previas. La misma puede ser medida por inmunoensayo high-performance liquid chromatography (HPLC); actualmente los métodos más utilizados en los países desarrollados son los enzimáticos. En estos momentos se necesita la estandarización de los métodos por la National Glycohemoglobin Standarization Program (NGSP) 14,15,16.

La HbA1c en el paciente con diabetes y enfermedad renal puede no indicar adecuadamente el control glucémico por las razones antes expuestas y también por la alteración de la vida media del eritrocito, anemia, ferropenia o el uso de eritropoyetina, entre otros. Algunos estudios han propuesto la medición de albumina glicada y fructosamina al atribuirles una menor variabilidad en la enfermedad renal avanzada, pero la escasa disponibilidad del método, la falta de experiencia y de estandarización no han generalizado su uso 17,18.

De todas maneras, la glucemia y $\mathrm{HbA} 1 \mathrm{c}$ aún son los métodos utilizados para el control glucémico. Al reconocer el medio interno del paciente portador de enfermedad renal crónica (ERC) muy variable, la interpretación correcta de estos valores debe realizarse al asumir clínicamente el grado de estabilidad 
para evitar la valoración de cifras únicas absolutas debiendo considerarse como un grupo de valores obtenidos en un tiempo, útiles para determinar la tendencia en la evolución del paciente.

Existen publicaciones sobre condiciones que modifican las mediciones de $\mathrm{HbA} 1$ que pueden dividirse en dos grupos ${ }^{19,20}$.

a) Condiciones que interfieren en la interpretación (no referidas a cambios en la glucemia plasmática)

- Factores fisiológicos: la edad (la HbA1c aumenta aproximadamente 0,1\% por cada década después de los 30 años) y la raza (los afroamericanos presentan niveles mayores de $\mathrm{HbA} 1 \mathrm{c}$ independiente del nivel de glucemia).

- Enfermedad renal crónica: debido a la sobrevida reducida de los eritrocitos y al uso de eritropoyetina con aumento el número de glóbulos rojos jóvenes se subestima el valor de $\mathrm{HbA} 1 \mathrm{c}$.

- Anemia ferropénica: aún sin anemia, la deficiencia de hierro subestima la HbA1c, así como también el tratamiento con hierro endovenoso.

- La vida media eritrocitaria: si la misma está disminuida los valores de HbA1c están subestimados.

- Glicosilación variable.

b) Factores que interfieren con la medición

- Uremia.

- Variantes de hemoglobina.

- Fármacos: altas dosis de aspirina y opioides, eritropoyetina o hierro.

- Triglicéridos aumentados en sangre.

\section{Fructosamina}

La medición de la fructosamina, menos costosa que la de $\mathrm{HbA} 1 \mathrm{c}$, no es válida en situaciones donde la albumina es menor a $3 \mathrm{mg} / \mathrm{dl}^{21}$.

La medición de fructosamina demostró bastante precisión en situaciones donde la $\mathrm{HbA1c}$ no refleja adecuadamente el estado glucémico ${ }^{22,23}$.

La combinación de la HbA1c junto con la fructosamina no es estadísticamente superior a la hemoglobina glicosilada sola. Su mayor limi- tación es la ausencia de evidencia fuerte en la asociación con las complicaciones crónicas de la diabetes, no siendo de utilidad para evaluar el control metabólico en pacientes con ERC ${ }^{24}$.

\section{Glucosuria}

No sería una herramienta de medición efectiva como el monitoreo del control glucémico en situaciones de enfermedad renal. Las razones por las que no se recomienda su realización rutinaria son:

1. El valor de la glucosa urinaria representa a todas las diversas glucemias que existieron durante el llenado de la vejiga o durante el intervalo entre el último vaciamiento vesical y la micción. Así, la valoración de la glucosuria no necesariamente corresponde a la glucemia del momento de la micción (evidencia nivel A).

2. No provee información sobre los niveles que se encuentran por debajo del umbral renal.

3. Existe una amplia variación individual del umbral renal para la glucosa. En pacientes con diabetes, el aumento de los cotransportadores $\mathrm{Na+-}$ glucosa (SGLT-2) en túbulo proximal y la elevación del umbral renal derivan en glucosuria que subestiman a la glucemia.

4. Un resultado negativo no discrimina entre hipoglucemia, normoglucemia e hiperglucemia leve o moderada.

5. Utiliza una escala visual de colores para comparar el resultado y tiene menor exactitud que el monitoreo de glucosa capilar que utiliza un sistema de lectura por glucómetro.

6. Se afecta por la ingesta de líquidos y la concentración de la orina.

7. Algunas drogas interfieren con las determinaciones.

8. Es impreciso a bajas concentraciones de glucosa, aunque los fabricantes especifiquen que son positivos con glucosurias $\geq 100 \mathrm{mg} / \mathrm{dl}$. La experiencia clínica y la investigación dan sustento a estos criterios, lo que desvaloriza su medición especialmente frente a la posibilidad del automonitoreo glucémico ${ }^{25}$.

\section{Cetonuria}

El deficiente aprovechamiento de la glu- 
cosa provoca lipólisis aumentada con la consecuente formación de ácidos grasos libres y coenzima $A$, parte de la cual se condensa formando ácido acético que es eliminado por riñón, reducido en hidroxibutírico y por decarboxilación en acetona. Estos tres compuestos aparecen en orina siendo la relación acetona/ácido acético 1/10, los dos últimos los únicos ácidos analizables en orina.

Tiene sensibilidad a partir de $5 \mathrm{mg} / \mathrm{dl}$. Su utilidad es limitada en pacientes con ERC y se emplea para prevenir complicaciones agudas relacionadas con la diabetes ${ }^{25}$.

\section{Automonitoreo glucémico}

El automonitoreo glucémico (AMG) pre y postprandial permite conocer las concentraciones de glucemia y el efecto del tratamiento sobre las mismas y posibilita identificar hipo/hiperglucemias al orientar los ajustes terapéuticos y proporcionar una retroalimentación más oportuna con respecto a los cambios de la medicación ${ }^{26}$.

En los pacientes que están en tratamiento con insulina se recomienda realizar tres o más automonitoreos glucémicos por día ${ }^{27}$. Quienes están en tratamiento intensificado con insulina o infusor de insulina deberían efectuar los controles preingestas, bed time, ocasionalmente postprandiales y en situaciones especiales (antes del ejercicio, luego de una hipoglucemia y previamente a realizar actividades como conducir automóviles) 5 .

En caso de pacientes con regímenes basal de insulina o agentes antidiabéticos orales es insuficiente la evidencia de cuándo indicar el AMG y la frecuencia dado que el tema es aún controvertido; de todas maneras, el AMG sería útil como guía para la adecuación del tratamiento, así como para obtener un buen control glucémico ${ }^{5}$.

Si se consideran los pacientes con diabetes y enfermedad renal avanzada, en estadios 5 o en terapia renal sustitutiva, deberían evaluarse todas las variables antes mencionadas; si se determina que no es buena la correlación entre la HbA1c y/o la fructosamina o albúmina glicadas con la glucemia, la única herramienta que permitiría conocer el perfil glucémico será entonces el automonitoreo. Posiblemente, a futuro, el monitoreo continuo será una herramienta útil para el reajuste del tratamiento, la evaluación de la variabilidad y las hipoglucemias, etc.

En conclusión, el AMG se indicará de manera individualizada según el tipo de diabetes, frecuencia de hipoglucemias, etapa de enfermedad renal, adherencia del paciente, tratamiento instituido y comorbilidades asociadas; también debe considerarse la capacidad de cada paciente para tomar decisiones una vez obtenido el resultado.

\section{BIBLIOGRAFÍA}

1. National Kidney Foundation. KDOQI. Clinical practice guidelines and clinical practice recommendations for diabetes and chronic kidney disease. A clinical update from KDIGO Kidney 2015; 87(1): 20-30

2. Akbari A, Clase CM, Acott $P$, et al. Canadian Society of Nephrology Commentary the KDIGO. Clinical practice guideline for CKD evaluation and management. Am J Kidney Dis. 2015; 65(2): 177-205.

3. Rohlfing $\mathrm{CL}$, Wiedmeyer HM, Little RR, et al. Defining the relationship between plasma glucose and $\mathrm{HbA}(1 \mathrm{c})$ : analysis of glucose profiles and $\mathrm{HbA}(1 \mathrm{c})$ in the Diabetes Control and Complications Trial. Diabetes Care. 2002; 25(2):275-278.

4. Stratton IM, Adler Al, Neil HA, et al. Association of glycaemia with macrovascular and microvascular complications of type 2 diabetes (UKPDS 35): prospective observational study. BMJ. 2000; 321(7258):405-412.

5. American Diabetes Association. Standards of Medical Care. Diabetes Care 2017; 40 (Suppl 1): S48-S56

6. American Diabetes Association. Standards of Medical Care. Diabetes Care 2012; 35 (Suppl 1):S11-S63.

7. Munshi MN, Segal AR, Suhl E, et al. Frequent hypoglycemia among elderly patients with poor glycemic control. Arch Intern Med 2011; 171(4):362-364.

8. Shurraw S, Hemmelgarn B, Lin M, et al. Association between glycemic control and adverse outcomes in people with diabetes mellitus and chronic kidney disease: a population based cohort study. Arch Intern Med 2011; 171: 1920-1927-

9. Ramirez SPB, McCullough KP, Thumma JR, et al. Hemoglobin A1c levels and mortality in the diabetic hemodialysis population: finding from Dialysis Outcomes and Practice Patterns Study (DOPPS) Diabetes Care 2012: 35:2527-2532. 
10. Duong U, Mehrotra R, Molnar MZ, et al. Glycemic control and survival in peritoneal dialysis patients with diabetes mellitus. Clin J A Nephrol 2011; 6(5):1041-1048.

11. Miller ME, Bonds DE, Gerstein $\mathrm{HC}$, et al. The effects of baseline characteristics, glycaemia treatment approach, and glycated haemoglobin concentration on the risk of severe hypoglycaemia: post hoc epidemiological analysis of the ACCORD study. BMJ 2010; 340:b5444.

12. Papademetriou V, Lovato L, Doumas $M$, et al. Chronic kidney disease and intensive glycemic control increase cardiovascular risk in patients with type 2 diabetes. Kidney Int 2015; 87:649-659.

13. Perkovic V, Heerspink HL, Chalmers J, et al. Intensive glucose control improves kidney outcomes in patients with type 2 diabetes. Kidney Int 2013; 83(3):517-523.

14. Goldstein DE, Little RR, Lorenz RA, et al.Tests of glycemia in diabetes. Diabetes Care 2004; 27 (7):1761-73 2004.

15. Little RR, Rohlfing CL, Wiedmeyer HM, et al. The national glycohemoglobin standardization program: a five year progress report. Clin Chem 2001; 47 (11):1985-92.

16. Little RR, Rohlfing CL, Sacks DB; National Glycohemoglobin Standardization Program (NGSP) Steering Committee. Status of hemoglobin A1c measurement and goals for improvement: from chaos to order for improving diabetes care. Clin Chem 2011; 57 (2):205-214.

17. Danese E, Montagnana M, Nouvenne A, et al. Advantages and pitfalls of fructosamine and glyacted albumin in diagnosis and treatment of diabetes. $\mathrm{J}$ of Diabetes Science and Technology 2015; 9(2) 169-176.

18. Inaba M, Okuno S, KumedaY, et al; Osaka CKD Expert Research Group. Glycated albumin is a better glycemic indicator than glycated hemoglobin values in hemodialysis patients with diabetes: effect of anemia and erythropoietin injection. J Am Soc Nephrol 2007; 18(3):896-903.
19. Dagogo-Jack S. Pitffals in the use of HbA1c as a diagnostic test: the ethnic conundrum. Nat Rev Endocrinol 2010; 6 (10):589-593.

20. Gallagher EJ, Le Roith D, Bloomgarden Z. Review of hemoglobin $A(1 \mathrm{c})$ in management of diabetes. J Diabetes 2009; 1 (1): 9-17.

21. Baker JR, O'Connor JP, Metcalf PA, Lawson MR, et al. Clinical usefulness of estimation of serum fructosamine concentration as a screening test for diabetes mellitus. Br Med J (Clin Res Ed) 1983 24; 287(6396):863-7.

22. Parrinello CM, Selvin E. Beyond $\mathrm{HbA} 1 \mathrm{c}$ and glucose: the role of nontraditional glycemic markers in diabetes diagnosis, prognosis, and management. Curr Diab Rep 2014; 14(11):548.

23. Selvin E, Rawlings AM, Grams M, et al. Fructosamine and glycated albumin for risk stratification and prediction of incident diabetes and microvascular complications: a prospective cohort analysis of the Atherosclerosis Risk in Communities (ARIC) study. Lancet Diabetes Endocrinol 2014;2 (4):279-288.

24. Sumner AE, Duong MT, Aldana PC, et al. A1C combined with glycated albumin improves detection of prediabetes in Africans: the Africans in America study. Diabetes Care 2016;39 (2):271-277.

25. Mainetti H. Laboratorio en diabetes mellitus. En: Ruiz M, Ruiz-Morosini ML (Editores) Diabetes Mellitus, Vol 1, $4^{\circ}$ Ed. Argentina Akadia; 2012, 215-218.

26. Poolsup N, Suksomboon N, Jiamsathit W. Systematic review of the benefits of self-monitoring of blood glucose on glycemic control in type 2 diabetes patients. Diabetes TechnolTher 2008; 10 (Suppl 1): S-51-S-66.

27. Standards of Medical Care in Diabetes, Position Statement, American Diabetes Association. Diabetes Care 2008; 31(Suppl1):S12-S54. 